\title{
A soft mechanical phenotype of SH-SY5Y neuroblastoma and primary human neurons is resilient to oligomeric $A \beta(1-42)$ injury
}

Terra M. Kruger ${ }^{1}$, Kendra J. Bell', Thiranjeewa I. Lansakara², Alexei V. Tivanski², Jonathan A. Doorn $^{1}$, Lewis L. Stevens ${ }^{1, *}$

1. Department of Pharmaceutical Sciences and Experimental Therapeutics, College of Pharmacy, The University of lowa, lowa City, IA 52242

2. Department of Chemistry, The University of lowa, lowa City, IA 52242

\section{Supporting Information}

\section{METHODS}

\section{$\underline{2 D}$ polyacrylamide gel preparation}

Polyacrylamide (PA) matrices with varying stiffness were prepared following the same protocol established in our previous publication. $18 \mathrm{~mm}$ round glass coverslips were coated with a uniform film of sodium hydroxide by evaporating a $500 \mu \mathrm{L}$ solution of $0.1 \mathrm{M} \mathrm{NaOH}$ on a hot plate set to $80^{\circ} \mathrm{C}$. The coverslips were silanated in a $1 \%$ aminopropyltriethoxysilane (APTES) solution for 10 minutes. After extensive washing with water, the coverslips were exposed to $0.5 \%$ glutaraldehyde for 30 minutes, then were dried with a Kimwipe. Glass slides were treated with $200 \mu \mathrm{L}$ of dichlorodimethylsilane (DCDMS), then washed extensively with water. The polyacrylamide gel solutions were made using varying solutions of Dulbeccos's phosphate buffered saline (DPBS), 2\% w/v N,N'-methylenebis(acrylamide) (BIS), and 40\% wt/vol acrylamide solutions. $40 \mu \mathrm{L}$ of the stock solutions were sandwiched between the silanated glass slide and the treated glass coverslip, and the solutions were allowed to polymerize for 30 minutes. The gels were transferred to a 12-well plate and sulfosuccinimidyl 6-(4'-azido2'nitrophenylamino) hexanoate (ThermoFisher) (Sulfo-SANPAH) was covalently cross-linked to the surface of the substrate using $1 \mathrm{~mL}$ per well of a $0.8 \mathrm{mg} / \mathrm{mL}$ solution under a UV light at $395 \mathrm{~nm}$ for 30 minutes. The gels were washed twice with $1 \mathrm{~mL}$ per well of $50 \mathrm{mM}$ HEPES buffer, and incubated with $10 \mu \mathrm{g} / \mathrm{mL}$ mouse collagen IV overnight at $4^{\circ} \mathrm{C}$. The coverslips were sterilized under a UV light in a biosafety cabinet and transferred to a new 12-well plate for cell seeding. The mechanical properties of these matrices were confirmed using the methods described in our previous studies ${ }^{2}$.

\section{AFM Nanoindentation}

For cells grown on PA matrices, each PA matrix was prepared as previously described, but on $35 \mathrm{~mm}$ glass coverslips, in $60 \mathrm{~mm}$ petri dishes. Either SH-SY5Y cells or primary neurons were plated onto the PA gels or collagen coated glass coverslips at $5 \times 10^{4}$ cells per petri dish. All force versus indentation depth data were collected using a Molecular force probe 3-D (Asylum Research, CA) AFM in a liquid cell. SH-SY5Y neuroblastoma or primary neurons were plated on either glass $35 \mathrm{~mm}$ coverslips or PA substrates, and were submerged in DPBS for the AFM measurements. Since AFM nanoindentation requires physical contact with the sample, cells suspended in the 3D collagen matrices were not evaluated for stiffness. Nanoindentation measurements were collected within an hour after submersion in the buffer solution. Two different cantilevers (Novascan, IA) were used that each had a polystyrene bead with radius of 
$2.25 \mu \mathrm{m}$ attached at the end of the cantilever for force measurements. The nominal spring constant was $0.06 \mathrm{~N} / \mathrm{m}$ and the actual spring constants were determined using a built-in thermal noise method. ${ }^{3}$ Individual cells were first located using the AFM top-view camera and force measurements were then collected at the approximate center of the cell. Force versus indentation depth curves were collected in the contact mode with maximum force range of 1-4 $\mathrm{nN}$ using a $0.7-1 \mu \mathrm{m} / \mathrm{s}$ tip approach velocity.

To determine the cell stiffness after exposure to oligomeric and fibrillated $\mathrm{A} \beta$, SH-SY5Y were plated onto $35 \mathrm{~mm}$ glass cover slips in $60 \mathrm{~mm}$ petri dishes in complete medium. The cell were treated with $1 \mu \mathrm{M}$ of either oligomeric or fibrillated $A \beta(1-42)$ for 24 hours, followed by cell stiffness measurements using AFM. The approach data of the force-indentation curves collected in buffer solution were fit to the Hertzian elastic contact model. ${ }^{1,4-5}$ For the Young's moduli calculations, a Poisson's ratio of 0.5 was used for the $\mathrm{SH}-\mathrm{SY} 5 \mathrm{Y}$ cells, primary neurons and polyacrylamide gels. ${ }^{6-}$ ${ }^{7}$ Approximately $20 \%$ of the force curves collected were excluded from the analysis due to probing on the edge of the cell. ${ }^{8}$ Higher stiffness yielding force curves are excluded from the analysis due to contribution of substrate effect which are a result of probing cells on the edge. For our cell stiffness results, averaged Young's moduli values were obtained by recording 10 consecutive measurements on each individual cell with a minimum of 12 different cells for each different matrix on which they were grown.

\section{AFM nanoindentation analysis}

AFM nanoindentation measurements were performed on individual cells to determine their stiffness. Approach force versus indentation depth data collected using nanoindentation measurements were fit to the simplified form of Hertzian elastic contact model given below to calculate stiffness of individual cells ${ }^{1}$;

$$
F=\frac{4 E^{*} \sqrt{R_{t i p}}}{3} h^{\frac{3}{2}}
$$

Where $F, R_{\text {tip }}$ and $h$ are the loading force, radius of curvature of colloidal AFM probe and indentation depth, respectively. $E^{*}$ is the reduced elastic modulus of the cells where it can be evaluated using following equation,

$$
\frac{1}{E^{*}}=\frac{1-v_{\text {tip }}^{2}}{E_{\text {tip }}}+\frac{1-v_{\text {sample }}^{2}}{E_{\text {sample }}}
$$

$\mathrm{E}_{\text {tip }}$ and $\mathrm{E}_{\text {sample }}$ are the Youngs modulus of the colloidal AFM probe and cell (either SH-SY5Y or PHN cells), $v_{\text {tip }}$ and $v_{\text {sample }}$ are Poisson's ratio of the probe and cell, respectively. Radius of colloidal AFM probe was estimated to be $2.25 \mu \mathrm{m} .{ }^{9-10}$ Data acquisition and analysis was carried out as reported in our previous work ${ }^{1,10}$. 
Figure S1. Representative AFM height images of fibrillated and oligomeric $A \beta$ drop casted on to an atomically flat mica surface (A) fibrillated CA, (B) oligomeric CA, (C) fibrillated APC and (D) oligomeric APC
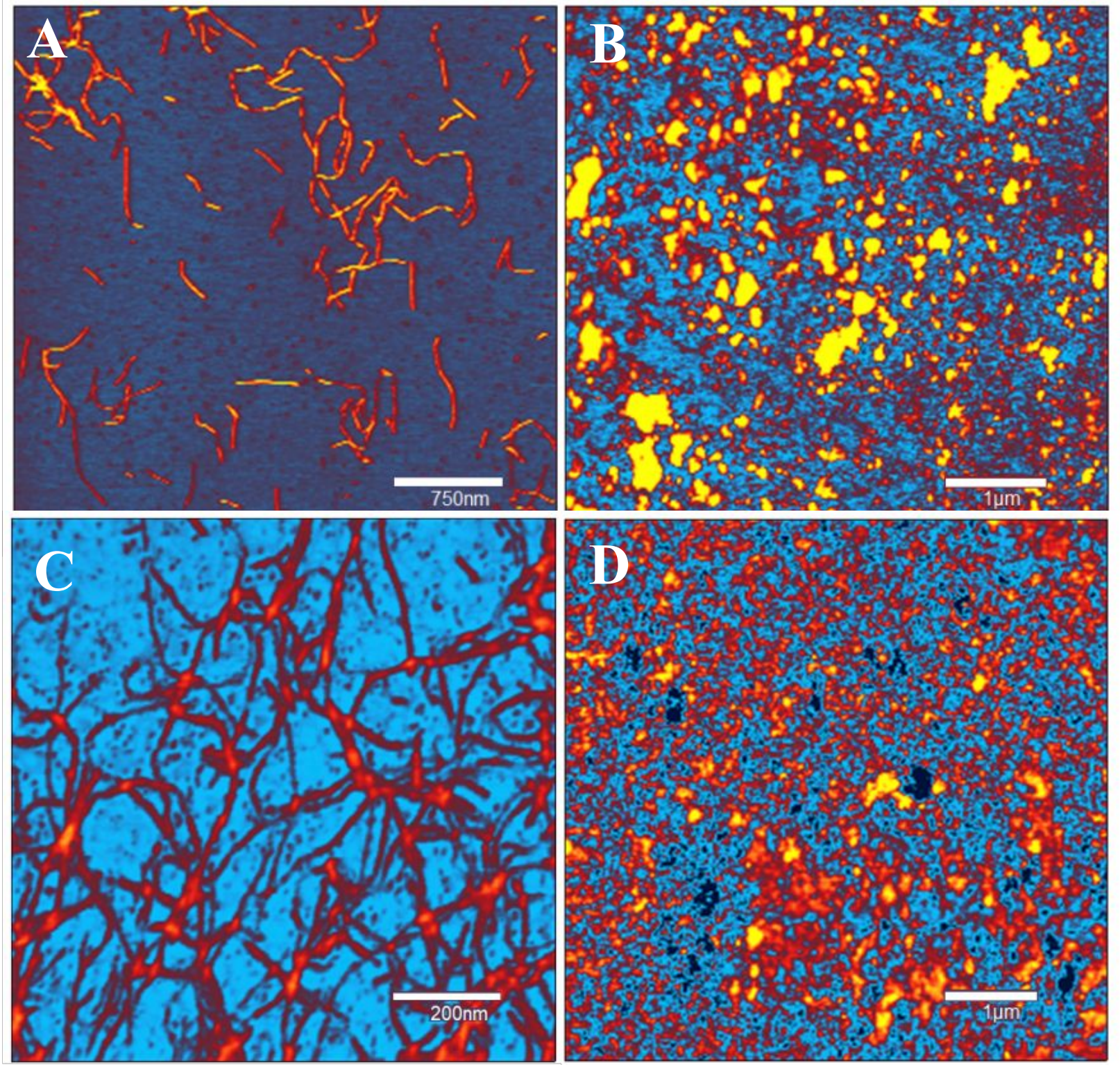
Figure S2. Representative force versus indentation depth profiles for individual cells (left panel untreated, fibrillated $A \beta$ dosed, oligomeric $A \beta$ dosed SH-SY5Y cells and right panel - undosed, fibrillated $A \beta$ dosed, oligomeric $A \beta$ dosed primary human neurons (PHN) cells) with the Hertzian elastic contact model fit. Red dots are data and black solid line is the fit.
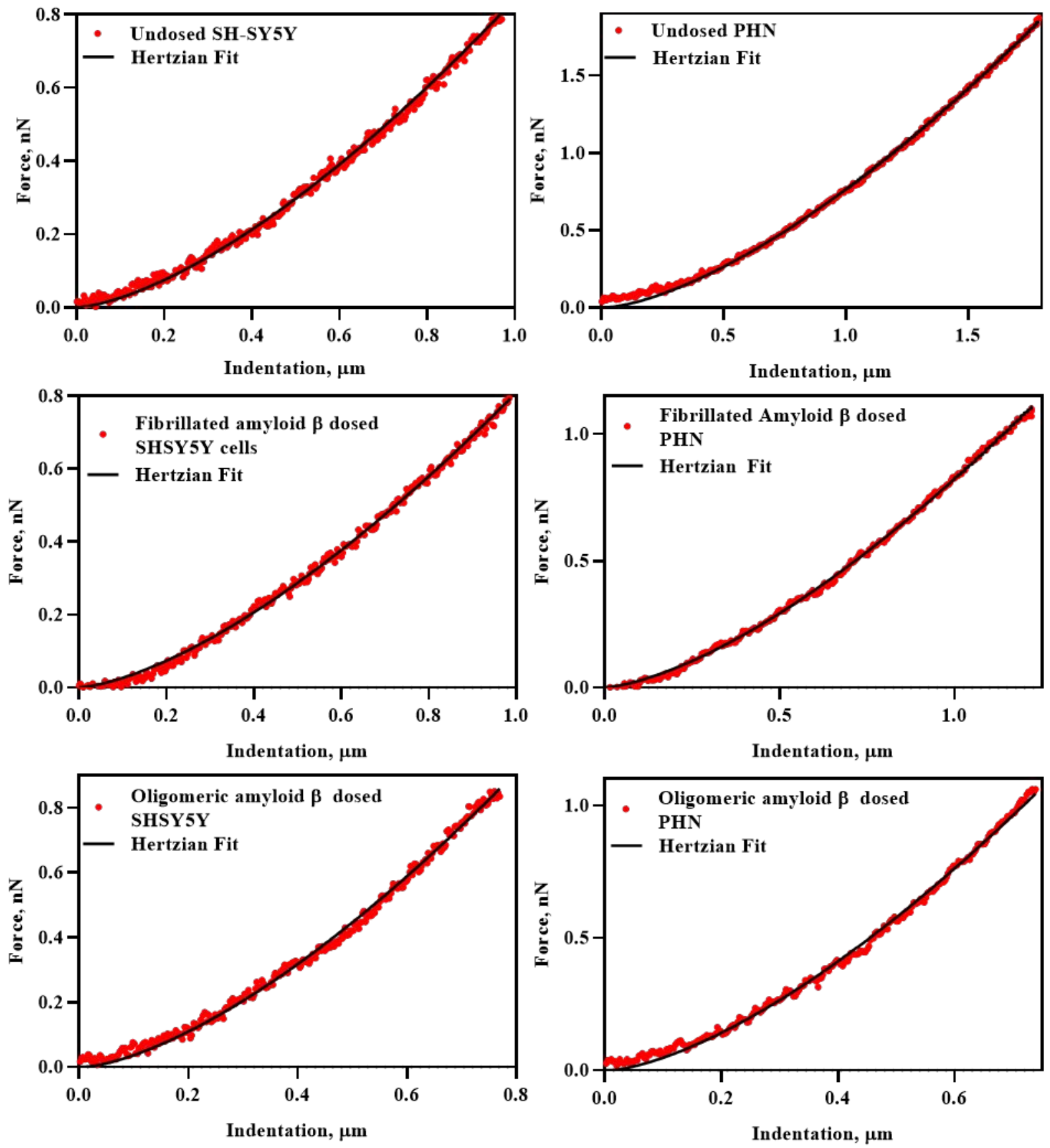
Figure S3. Representative force versus indentation depth profiles for individual primary human neurons plated on different PA matrices.
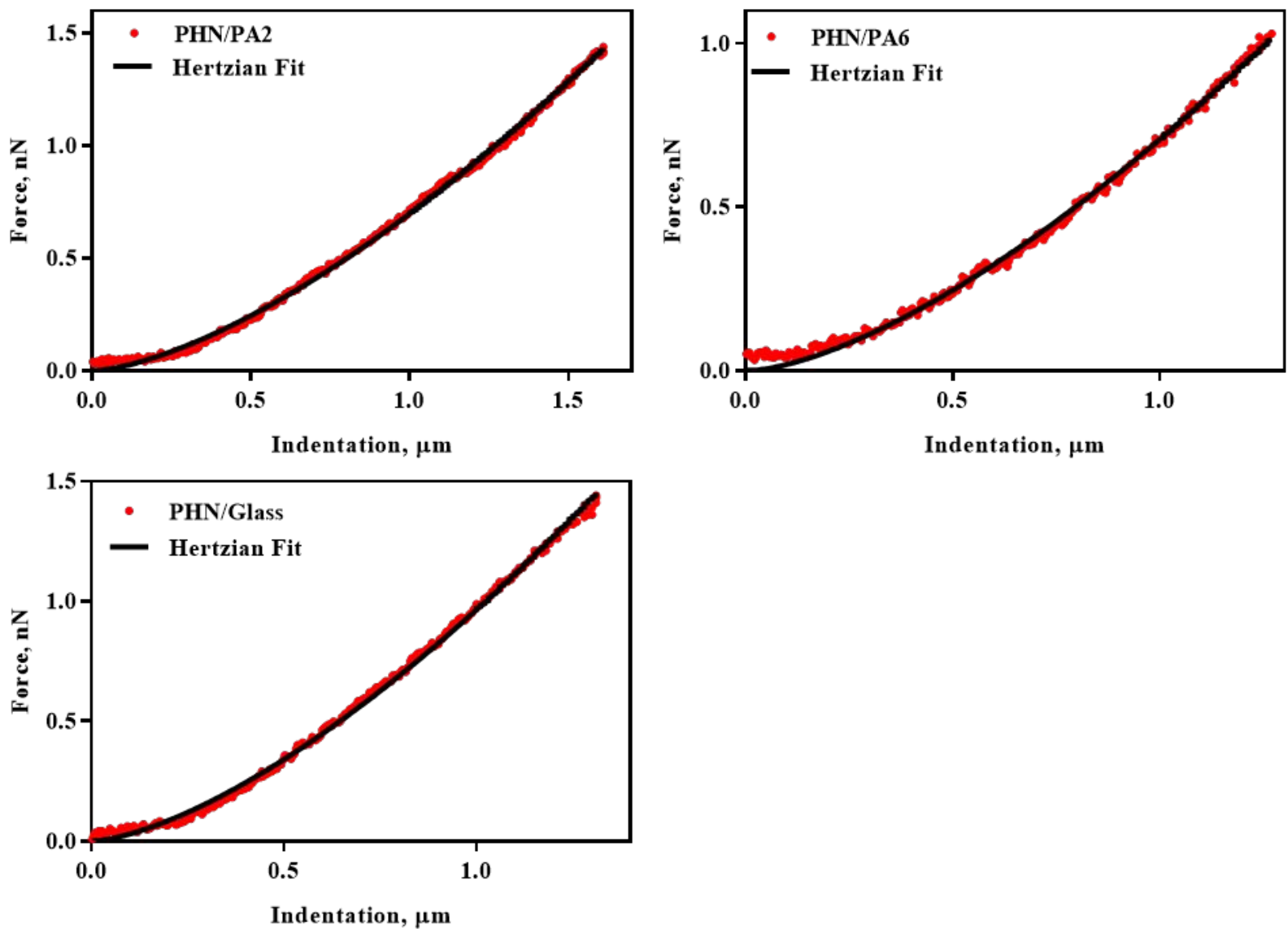

Figure S4. Representative force versus indentation depth profiles for bare PA matrices (PA2, soft) and (PA6, stiff).
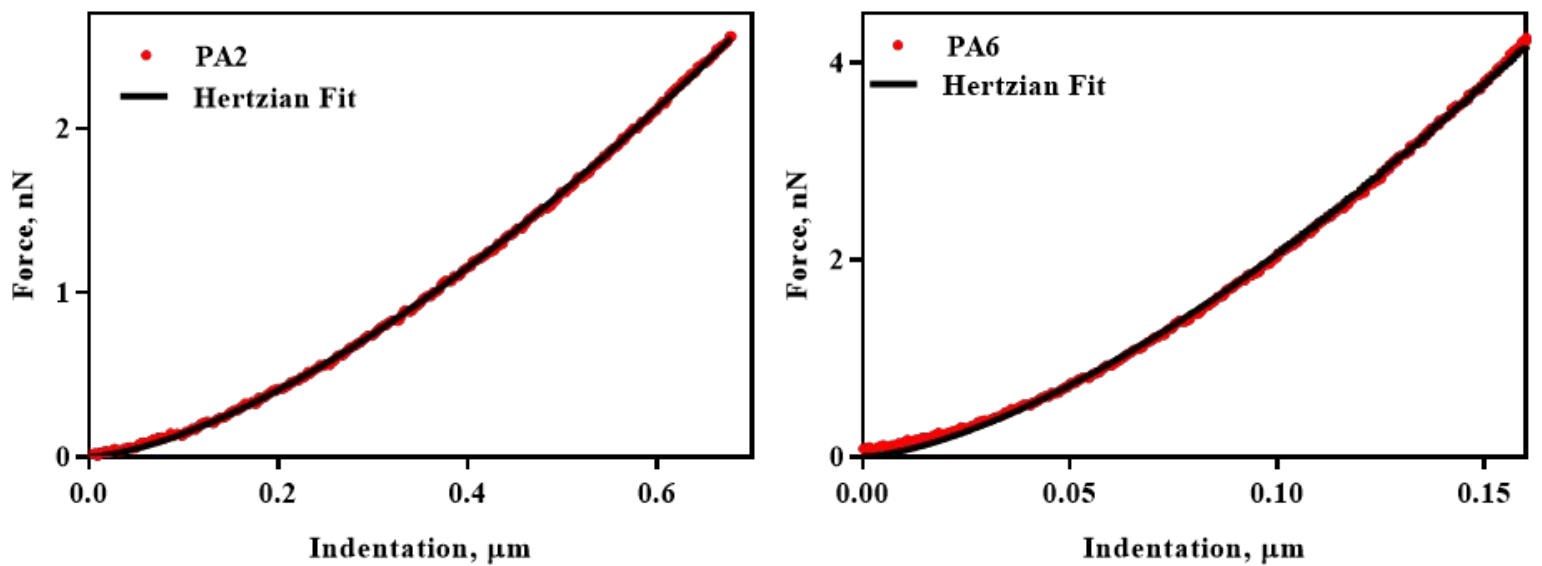
Figure S5. Quantitation of cell area for primary human cortical neurons which show an increase in cell area with increasing substrate stiffness (One-way ANOVA, $p<0.05$ ).

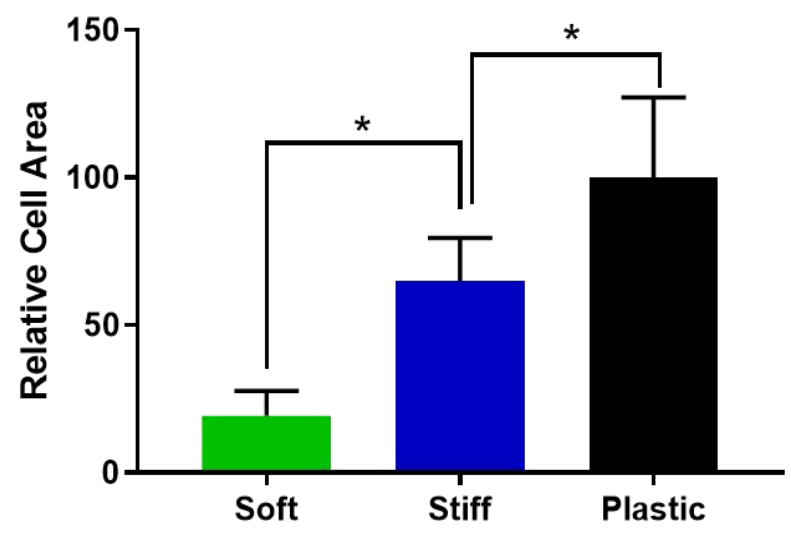

Figure S6. Shear modulus of 3D collagen matrices prepared at two different concentrations.

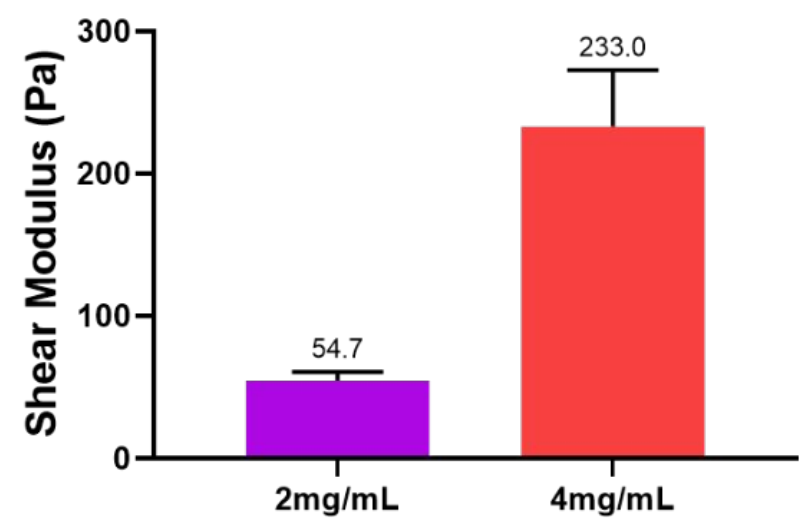




\section{Oligomeric $A \beta(1-42)$ uptake studies}

\section{Preparation of fluorescent oligomeric species}

HFIP treated Abeta was dissolved in $2 \mu \mathrm{L}$ of DMSO. To this solution, $98 \mu \mathrm{L}$ of sterile DMEM was added for a final $A \beta$ concentration of $100 \mu \mathrm{M}$. This was combined with $50 \mu \mathrm{g}$ of FITCtagged Abeta that had also been dissolved in $2 \mu \mathrm{L}$ of DMSO and then mixed with $98 \mu \mathrm{L}$ of sterile DMEM. The final solution was vortexed in a black microcentrifuge tube and refrigerated overnight.

\section{Flow cytometry}

Cells were grown on gels or plastic in a 12-well plate dosed with $1 \mu \mathrm{M}$ oligomeric Abeta for 24 hours. The cells were then trypsinized by first washing all samples with PBS and then placing the coverslips upside-down in $100 \mu \mathrm{L}$ of trypsin or by adding $100 \mu \mathrm{L}$ of trypsin to the cells plated on plastic. The plates were put onto a shaker table for 15 minutes. The cells were then transferred into a microcentrifuge tube along with $200 \mu \mathrm{L}$ of complete media. The tubes were centrifuged at $1.2 \mathrm{~g}$ for 5 minutes and the liquid was then aspirated, leaving the pellet behind. Then, $300 \mu \mathrm{L}$ of media was added to resuspend each pellet. The samples were then filtered into flow cytometry tubes placed in ice. Flow cytometry was performed on a FACScan 200 Flow cytometer. All uptake experiments were done in triplicate with 10,000 cells in each analysis.

Figure S7. Uptake of oligomeric $A \beta(1-42)$ by a.) SH-SY5Y and b.) human primary cortical neurons as a function of different substrate types: traditional cell culture "plastic", "soft" 2D PA matrix and a "stiff" 2D PA matrix.
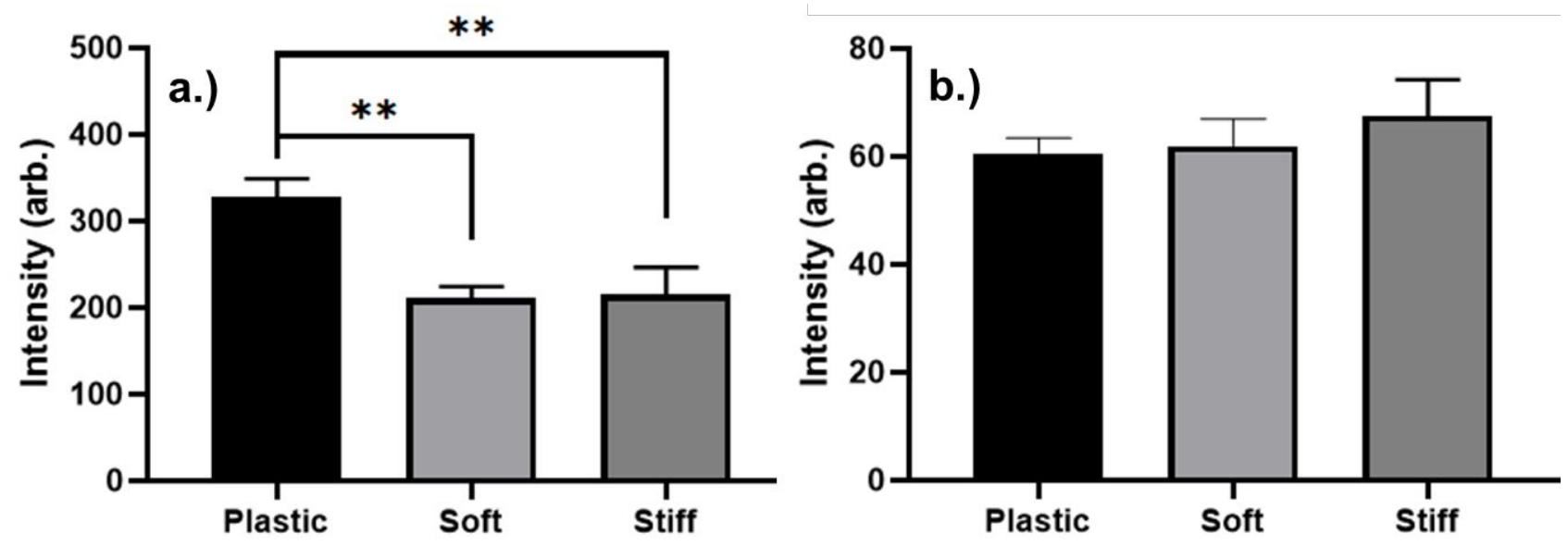
Figure S8. Viability of SH-SY5Y upon exposure to manganese chloride and oligomeric A $\beta$. Pretreatment with $10 \mu \mathrm{M} \mathrm{MnCl}_{2}$ agonizes integrin activation to simulate higher cell adhesion. This resulted in a significant decrease in MTS viability relative to cells exposed to $5 \mu \mathrm{M} A \beta$ alone.

(Two-tailed t-test, $p<0.05$ ) Under the same conditions, the human primary neurons displayed no change in viability between pre-treated $+A \beta$ and $A \beta$ alone groups.

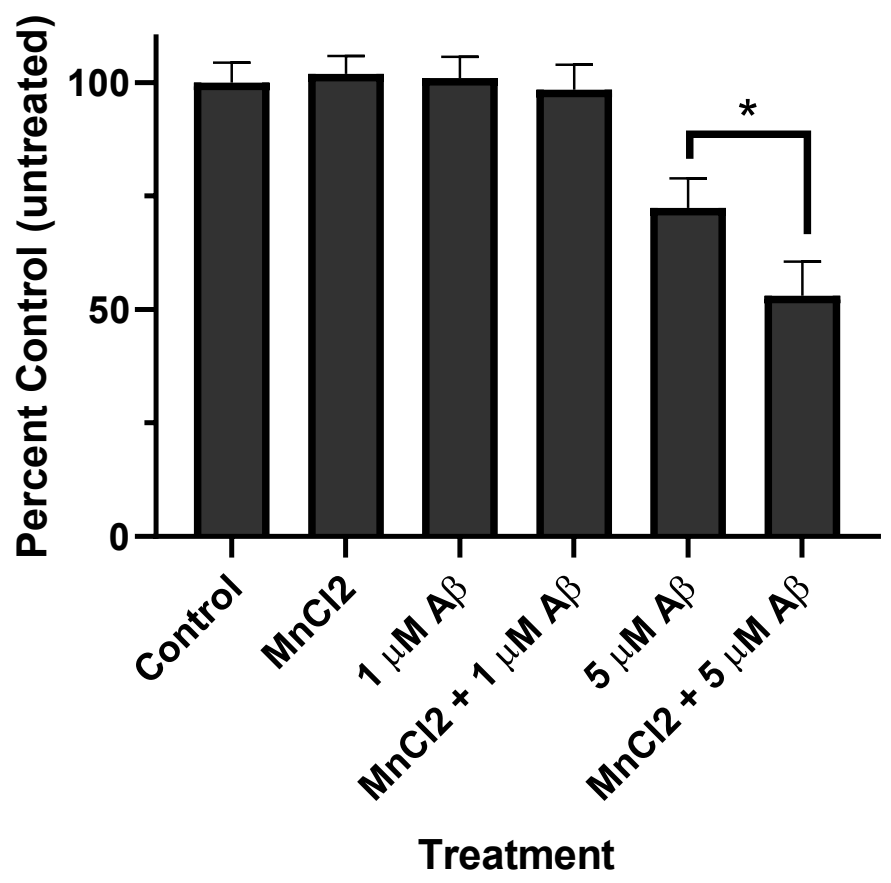




\section{REFERENCES}

1. Kruger, T. M.; Bell, K. J.; Lansakara, T. I.; Tivanski, A. V.; Doorn, J. A.; Stevens, L. L., Reduced extracellular matrix stiffness prompts $\mathrm{SH}-\mathrm{SY} 5 \mathrm{Y}$ cell softening and actin turnover to selectively increase Abeta(1-42) endocytosis. ACS Chem Neurosci 2019, 10 (3), 1284-1293.

2. Hutter, J. L.; Bechhoefer, J., Calibration of atomic-force microscope tips. Rev Sci Instrum 1993, 64 (7), 1868-1873.

3. Rupasinghe, T. P.; Hutchins, K. M.; Bandaranayake, B. S.; Ghorai, S.; Karunatilake, C.; Bučar, D.K.; Swenson, D. C.; Arnold, M. A.; MacGillivray, L. R.; Tivanski, A. V., Mechanical properties of a series of macro- and nanodimensional organic cocrystals correlate with atomic polarizability. Journal of the American Chemical Society 2015, 137 (40), 12768-12771.

4. Scarcelli, G.; Polacheck, W. J.; Nia, H. T.; Patel, K.; Grodzinsky, A. J.; Kamm, R. D.; Yun, S. H., Noncontact three-dimensional mapping of intracellular hydromechanical properties by Brillouin microscopy. Nat Methods 2015, 12 (12), 1132-4.

5. Guz, N.; Dokukin, M.; Kalaparthi, V.; Sokolov, I., If Cell Mechanics Can Be Described by Elastic Modulus: Study of Different Models and Probes Used in Indentation Experiments. Biophysical Journal 2014, 107 (3), 564-575.

6. Krymskaya, K. I.; Andreeva, N. V.; Filimonov, A. V., A technique for determining Young's modulus of biological objects using atomic-force microscopy in the wide temperature range below Rt. St. Petersburg Polytechnical University Journal: Physics and Mathematics 2016, 2 (3), 217-223.

7. Solon, J.; Levental, I.; Sengupta, K.; Georges, P. C.; Janmey, P. A., Fibroblast Adaptation and Stiffness Matching to Soft Elastic Substrates. Biophysical Journal 2007, 93 (12), 4453-4461.

8. $\quad$ Kruger, T. M.; Givens, B. E.; Lansakara, T. I.; Bell, K. J.; Mohapatra, H.; Salem, A. K.; Tivanski, A. V.; Stevens, L. L., Mechanosensitive Endocytosis of High-Stiffness, Submicron Microgels in Macrophage and Hepatocarcinoma Cell Lines. ACS Applied Bio Materials 2018, 1 (5), 1254-1265.

9. $\quad$ Kruger, T. M.; Bell, K. J.; Lansakara, T. I.; Tivanski, A. V.; Doorn, J. A.; Stevens, L. L., Reduced Extracellular Matrix Stiffness Prompts SH-SY5Y Cell Softening and Actin Turnover To Selectively Increase A $\beta(1-42)$ Endocytosis. ACS Chemical Neuroscience 2018.

10. Mohapatra, H.; Kruger, T. M.; Lansakara, T. I.; Tivanski, A. V.; Stevens, L. L., Core and surface microgel mechanics are differentially sensitive to alternative crosslinking concentrations. Soft Matter 2017, 13 (34), 5684-5695. 